\title{
Doxycycline-Induced Acute Pancreatitis: A Rare Adverse Event
}

\author{
Prashanth Rawla ${ }^{a, c}$, Jeffrey Pradeep Raj ${ }^{b}$
}

\begin{abstract}
Doxycycline is a broad-spectrum antibiotic belonging to the tetracycline group which acts by inhibiting bacterial protein synthesis. It is considered to be a relatively safe drug. We report a case of doxycycline-induced acute pancreatitis (DIAP) in an adult female patient who was started on the usual therapeutic dose 1 week before for acne vulgaris. The WHO causality assessment was possible, and the Naranjo scale confirmed it as "definite" adverse drug reaction. A brief literature review on case reports previously reporting DIAP has also been summarized.
\end{abstract}

Keywords: Doxycycline; Pancreatitis; Drug-induced pancreatitis

\section{Introduction}

Doxycycline is a broad-spectrum antibiotic belonging to the antibiotic drug class of tetracyclines which are bacteriostatic to a broad spectrum of both aerobic and anerobic gram-positive and gram-negative bacteria. It acts by inhibiting the bacterial protein synthesis by binding to the $30 \mathrm{~S}$ subunit of the bacterial ribosome and preventing the binding of aminoacyl-tRNA to the acceptor site on the mRNA-ribosome complex. Doxycycline is unique in the sense that it does not undergo metabolism and is excreted unchanged in the urine and bile. It also does not require a dose modification in renal impairment. The common side effects of tetracycline groups of drugs are gastrointestinal side effects and bone and teeth deposits. Whereas the less common ones include photosensitivity, renal toxicity and hepatic toxicity in patients with impaired kidney functions. These side effects are even less frequent in patients who use newer tetracycline like the doxycycline, tigecycline and minocycline [1]. Here we present an interesting case of a female adult patient who was diagnosed with doxycycline-induced acute pancrea-

Manuscript submitted April 10, 2017, accepted April 25, 2017

aDepartment of Internal Medicine, Monmouth Medical Center, Long Branch, NJ, USA

bDepartment of Pharmacology, St John's Medical College, Bangalore, India ${ }^{\mathrm{c} C}$ Corresponding Author: Prashanth Rawla, Department of Internal Medicine, Monmouth Medical Center, Long Branch, NJ, USA.

Email: rawlap@gmail.com

doi: https://doi.org/10.14740/gr838w titis (DIAP).

\section{Case Report}

A 52-year-old adult female who is a known patient of fibromyalgia and spondylolisthesis at the lumbar vertebral levels L2, 3, 4 for the last 3 years presented with a 3-day history of acute onset very severe epigastric pain increasing in severity with time since onset. At presentation, the patient reported a score of 10 on a pain rating scale of 10 . The quality of the pain was sharp, and it was radiating to the back with mild relief on bending forward. There were no known aggravating factors. The pain was associated with nausea and non-bilious vomiting with no traces of blood. The patient denied any similar episodes in the past and has no history of alcohol consumption. She has a history of cholecystectomy done 2 years back for abdominal pain, but the gallbladder was apparently devoid of any gallstones.

An ultrasound and a computed tomography of the abdomen and blood workup were performed to determine the cause of pain. At admission, the serum lipase levels were elevated to $>1,600 \mathrm{IU} / \mathrm{L}$ (laboratory reference level 10 - $53 \mathrm{IU} / \mathrm{L}$ ). An ul-

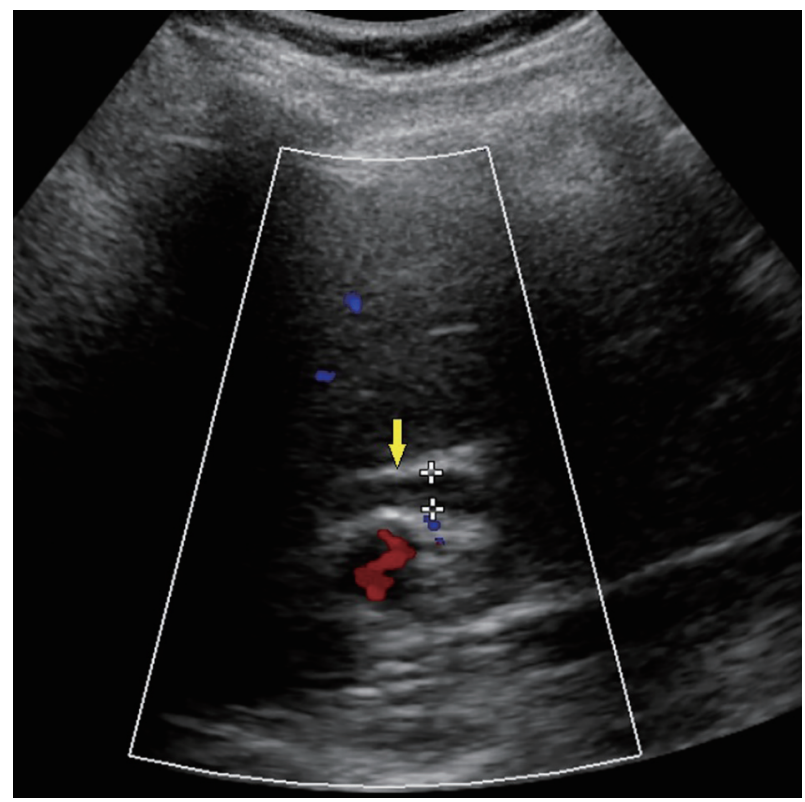

Figure 1. Ultrasound abdomen done on day 1. Yellow arrow shows common bile duct diameter within normal limits of $5.4 \mathrm{~mm}$. 


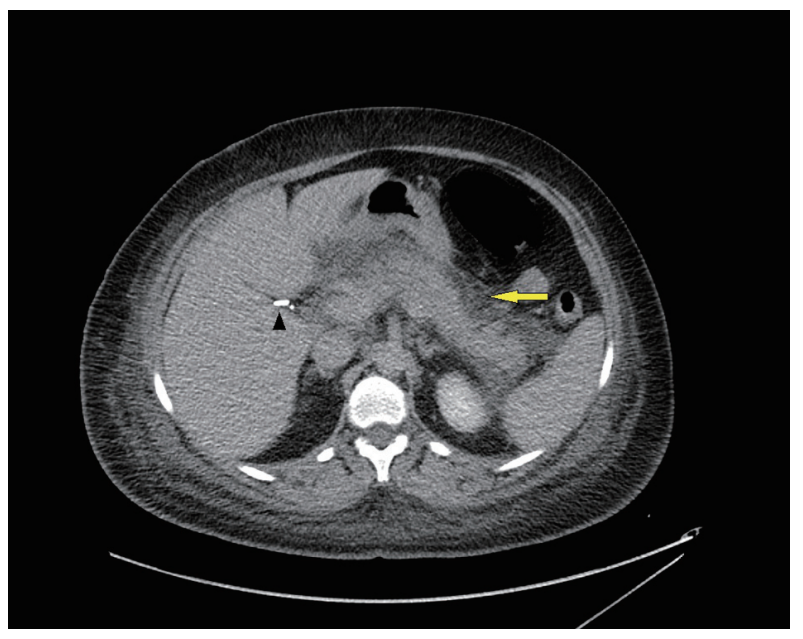

Figure 2. Computed tomography of the abdomen done on day 1. Yellow arrow shows edema in body of pancreas with pancreatic fluid and stranding consistent with acute pancreatitis. Black arrow head shows prior cholecystectomy.

trasound of the abdomen revealed a normal common bile duct with its diameter of $5.4 \mathrm{~mm}$ (Fig. 1). The abdomen CT findings were also consistent with the diagnosis of acute pancreatitis wherein there was stranding in the retroperitoneum and the abdominal mesentery with trace fluid in the perihepatic and pelvic space. There was edema of the head and body of the pancreas with peripancreatic edema but without any pancreatic pseudocyst. The remaining findings were unremarkable with no intrahepatic or extrahepatic biliary dilatation, patent mesenteric blood vessels and normal spleen and adrenal glands (Fig. 2). Further workup was done to look for other less frequent causes of pancreatitis. A magnetic resonance cholangiopancreatography (MRCP) was done which did not reveal any gallstones or sludge in the common bile duct (Fig. 3). IgG4 levels were normal thus ruling out autoimmune pancreatitis. Triglycerides were within the normal limits, and viral hepatitis panel was negative. At this stage, a possibility of drug-induced acute pancreatitis was considered. The patient was on oxycodone $5 \mathrm{mg}$ twice daily for fibromyalgia and gabapentin $600 \mathrm{mg}$ three times daily for neuropathic pain for the last 3 years. She also gave a recent history of being prescribed doxycycline 100 mg twice daily by her dermatologist for worsening acne. The patient had been taking doxycycline regularly for the past 7 days. Since the patient was doing all right with oxycodone and gabapentin for a longer time, it was considered that doxycycline would be the most probable cause for the patient's condition. However, all three drugs were stopped immediately. By then, the serum lipase levels began to fall. It was $1,140 \mathrm{IU} / \mathrm{L}$ the second day, $347 \mathrm{IU} / \mathrm{L}$ the third day and $111 \mathrm{IU} / \mathrm{L}$ on day 4.

The patient was started on enteral feeding when her nausea subsided. However, her abdominal pain persisted. Due to her constant and severe abdominal pain, a repeat abdomen CT was done on day 6 which showed a little necrosis of $6 \mathrm{~mm}$ in the head of the pancreas. The patient was managed conservatively with IV fluids and parenteral morphine. After the repeat abdomen CT was done, the patient was rechallenged with oxycodone and gabapentin, and the lipase levels were found to be

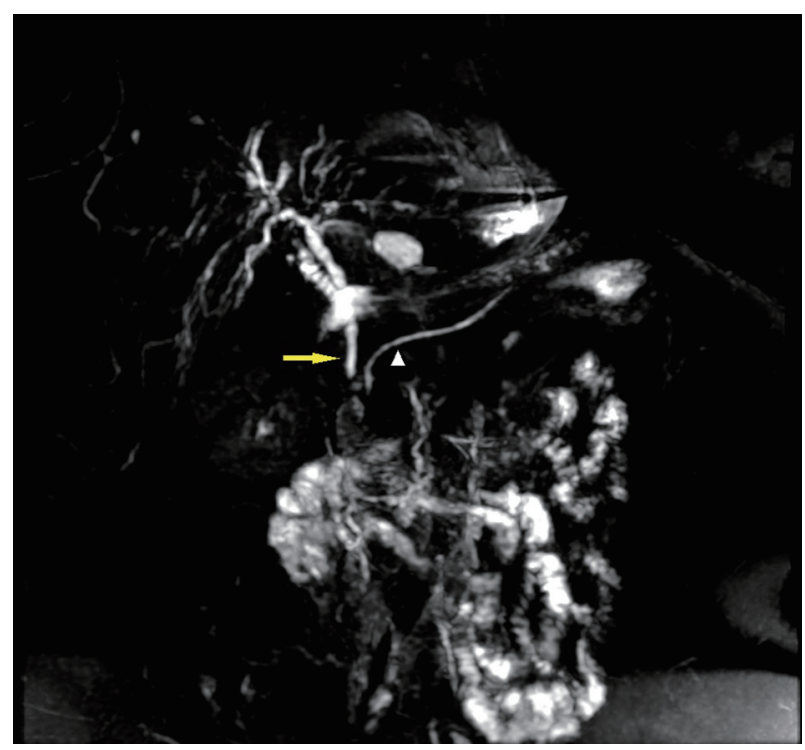

Figure 3. Magnetic resonance cholangiopancreatography done on day 2. Yellow arrow shows common bile duct measures $5.5 \mathrm{~mm}$ in diameter, no CBD stones. White arrow head shows no obstruction in pancreatic duct.

normal. As the patient's condition improved, oral feeding was started and was subsequently discharged.

\section{Discussion}

Acute pancreatitis is a life-threatening condition with an incidence of 45 per 100,000 people annually [2]. Drug-induced pancreatitis (DIP) is a very rare entity. The estimated incidence ranges from $0.1 \%$ to $5.3 \%$ in human immunodeficiency negative individuals [3, 4]. A retrospective study from Germany in 1993 reported the incidence of DIP to be $1.4 \%$ [5]. The study from the Czech Republic in 2010 reported that among 170 cases of acute pancreatitis, 5.3\% were DIP [4]. Thus, the exact incidence of DIP is very hard to determine, and of this, doxycycline to be reported as a causative drug has been exceedingly rare [6]. A PubMed search which was performed using the MeSH terms "Doxycycline" AND "Pancreatitis" shortlisted just two case reports reporting definite DIAP [6, 7]. There were two other case reports reporting probable DIAP [8, 9]. The case reported by Ocal et al [8] had concomitant use of ornidazole and a much higher dose of doxycycline $(500 \mathrm{mg}$ twice daily). While the other case reported by Moy and Kapila [9] had other causes of pancreatitis namely highly uncontrolled diabetes mellitus (HbA1c: 15.5\%) and diabetic ketoacidosis which are more common than DIP. Thus, it is evident that DIAP is a rare condition.

The diagnosis of DIAP was a diagnosis of exclusion after all other common causes such as biliary disease and alcoholism were ruled out. In our patient, there were few more concomitant drugs used for other co-morbidities. Since the patient was on these medications for more than 3 years, it was unlikely that they caused pancreatitis. However, these drugs were also stopped and later rechallenged after 6 days of stopping which 
proved harmless as confirmed by the normal serum lipase levels. Hence we believe that our case is one of the well-proven cases of DIAP with a well corroborating temporal association of the use of a drug. The mechanism by which it causes pancreatitis is largely unknown. However, because of the rarity, it may be considered as an idiosyncratic reaction [6].

According to the WHO causality assessment, doxycycline is the "probable" cause for DIP as de-challenge was positive and re-challenge was not done. According to the Naranjo algorithm, the adverse drug reaction (ADR) was classified as "definite" ADR with a score of 9 ( 2 points each for an adverse event occurring after doxycycline was administered and other causes being ruled out. One point each for the availability of previous conclusive reports of DIAP, an improvement on stopping the drug, less severe when the dose was decreased, and pancreatitis confirmed objectively with imaging and blood tests) [10]. According to the Schumock and Thornton preventability scale, the ADR was unpreventable [11] and based on the Hartwig and Siegel severity assessment scale, the severity of the reaction is placed at level 5 which involves withholding of the suspected drug and a prolonged hospital stay by at least 1 day with admission in the intensive care unit [10].

\section{Conclusion}

Doxycycline is a well-studied drug with a good safety profile, and food and drug administration (FDA) approved it for multidisciplinary clinical uses like the treatment of a wide variety of bacterial infections including acne vulgaris and periodontitis. Here, we report a rare case of DIAP. It is important that the physician keeps such rare adverse events in mind and have a high degree of suspicion to tackle the crisis effectively. To conclude, drug should be entertained as a possible etiology of idiopathic pancreatitis when other common causes are ruled out keeping in mind the vastly expanding pharmacotherapy in the recent times.

\section{Funding Support}

This research received no specific grant from any funding agency in the public, commercial, or not-for-profit sectors.

\section{Conflicts of Interest}

The authors do not have any conflicts of interest to disclose.

\section{Financial Disclosure}

None.

\section{Author Contributions}

Study design, drafting, critical revisions and final approval by PR and JPR.

\section{References}

1. Rang HP, Ritter JM, Flower RJ, Henderson G. Rang \& Dales Pharmacology. 8th ed. Elsevier Ltd: 2016; pp 632634.

2. Yadav D, Lowenfels AB. The epidemiology of pancreatitis and pancreatic cancer. Gastroenterology. 2013;144(6):1252-1261.

3. Balani AR, Grendell JH. Drug-induced pancreatitis : incidence, management and prevention. Drug Saf. 2008;31(10):823-837.

4. Vinklerova I, Prochazka M, Prochazka V, Urbanek K. Incidence, severity, and etiology of drug-induced acute pancreatitis. Dig Dis Sci. 2010;55(10):2977-2981.

5. Lankisch PG, Droge M, Gottesleben F. Drug induced acute pancreatitis: incidence and severity. Gut. 1995;37(4):565567.

6. Inayat F, Virk HU, Yoon DJ, Riaz I. Drug-Induced Pancreatitis: A Rare Manifestation of Doxycycline Administration. N Am J Med Sci. 2016;8(2):117-120.

7. Wachira JK, Jensen CH, Rhone K. Doxycycline-induced pancreatitis: a rare finding. S D Med. 2013;66(6):227229.

8. Ocal S, Selcuk H, Korkmaz M, Unal H, Yilmaz U. Acute pancreatitis following doxycycline and ornidazole coadministration. JOP. 2010;11(6):614-616.

9. Moy BT, Kapila N. Probable doxycycline-induced acute pancreatitis. Am J Health Syst Pharm. 2016;73(5):286291.

10. Srinivasan R, Ramya G. Adverse Drug Reaction-Causality Assessment. Int J Res Pharm Chem. 2011;1(3):606612.

11. Schumock GT, Thornton JP. Focusing on the preventability of adverse drug reactions. Hosp Pharm. 1992;27(6):538. 